\title{
Atividade antioxidante de extratos de Myrciaria dubia (camu-camu) Myrtaceae
}

\author{
Antioxidant activity of Myrciaria dubia (camu-camu) extracts Myrtaceae \\ Actividad antioxidante de los extractos de Myrciaria dubia (camu-camu) Myrtaceae
}

Recebido: 25/12/2021 | Revisado: 31/12/2021 | Aceito: 16/01/2022 | Publicado: 18/01/2022

\author{
Carlena Sinara Martins da Silva \\ ORCID: https://orcid.org/0000-0002-1543-8334 \\ Universidade Federal do Pará, Brasil \\ Universidade Federal do Oeste do Pará, Brasil \\ Centro Universitário da Amazônia, Brasil \\ E-mail: karlena_sinara@hotmail.com \\ Rosa Helena Veras Mourão \\ ORCID: https://orcid.org/0000-0003-0100-644X \\ Universidade Federal do Oeste do Pará, Brasil \\ E-mail: mouraorhv@yahoo.com.br
}

\begin{abstract}
Resumo
O fruto de Myrciaria dubia é rico em bioativos, este apresenta alto teor de ácido ascórbico e compostos fenólicos, por este motivo, o fruto vem ganhando destaque pela sua capacidade antioxidante. O objetivo deste estudo foi avaliar se diferentes extratos obtidos de cascas do fruto de $M$. dubia influencia na atividade antioxidante. Extratos hidroalcoólico $70 \%$ (EHA) e glicólicos 10 e 20\% (EG10 e EG20) foram obtidos de cascas a partir de um pó moderadamente grosso com perda por dessecação de $13,53 \%$, cinzas totais de $1,95 \%$, cinzas insolúveis em ácidos de $0,057 \%, \mathrm{pH} 2,53 \pm 0,05$ e índice de intumescência de $1,1 \mathrm{ml}$. Os extratos foram caracterizados quanto ao teor de ácido ascórbico e polifenóis totais e a atividade antioxidante determinada por DPPH e ABTS ${ }^{+}$. O EHA apresentou $7011 \pm 39 \mathrm{mg} / 100 \mathrm{ml} \mathrm{de} \mathrm{ácido}$ ascórbico e 7,75\% de polifenóis totais, enquanto que, nos EG10 e EG20 o teor de ácido ascórbico foi $3034 \pm 0$ e $6137 \pm 0 \mathrm{mg} / 100 \mathrm{~mL}$ e de polifenóis totais de 2,65 e $3,55 \%$ respectivamente. Os extratos apresentaram capacidade

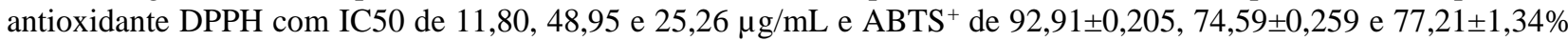
nos extratos EHA, EG10 e EG20, respectivamente. Os extratos apresentam conteúdos importantes de compostos bioativos, porém, o solvente influenciou no conteúdo e na atividade biológica, sendo o EHA com maior teor de bioativos o que refletiu também na sua maior capacidade antioxidante.
\end{abstract}

Palavras-chave: Camu-camu; Antioxidante; Extrato hidroalcoólico; Extrato glicólico.

\begin{abstract}
The fruit of Myrciaria dubia is rich in bioactives, these have a high content of ascorbic acid and phenolic compounds, for this reason, the fruit has been gaining prominence for its antioxidant capacity. The aim of this study was to evaluate whether different extracts obtained from M. dubia fruit peels influence the antioxidant activity. $70 \%$ hydroalcoholic (EHA) and 10 and 20\% glycolic extracts (EG10 and EG20) were obtained from bark from a moderately coarse powder with loss on desiccation of $13.53 \%$, total ash of $1.95 \%$, ash insoluble in acids of $0.057 \%$, $\mathrm{pH} 2.53 \pm 0.05$ and swelling index of $1.1 \mathrm{ml}$. The extracts were characterized as to the content of ascorbic acid and total polyphenols and the antioxidant activity determined by DPPH and ABTS+. The EHA showed $7011 \pm 39 \mathrm{mg} / 100 \mathrm{ml}$ of ascorbic acid and $7.75 \%$ of total polyphenols, while in the EG10 and EG20 the ascorbic acid content was $3034 \pm 0$ and $6137 \pm 0 \mathrm{mg} / 100 \mathrm{~mL}$ and total polyphenols of 2.65 and $3.55 \%$ respectively. The extracts showed DPPH antioxidant capacity with IC50 of $11.80,48.95$ and $25.26 \mu \mathrm{g} / \mathrm{mL}$ and ABTS+ of $92.91 \pm 0.205,74.59 \pm 0.259$ and $77.21 \pm 1.34 \%$ in the EHA extracts, EG10 and EG20, respectively. The extracts have important contents of bioactive compounds, however, the solvent influenced the content and biological activity, with EHA having the highest content of bioactives, which also reflected in its greater antioxidant capacity.
\end{abstract}

Keywords: Camu-camu; Antioxidant; Hydroalcoholic extract; Glycolic extract.

\section{Resumen}

El fruto de Myrciaria dubia es rico en bioactivos, estos tienen un alto contenido en ácido ascórbico y compuestos fenólicos, por ello, el fruto ha ido ganando protagonismo por su capacidad antioxidante. El objetivo de este estudio fue evaluar si diferentes extractos obtenidos de la cáscara del fruto de $M$. dubia influyen en la actividad antioxidante. Se obtuvieron extractos hidroalcohólicos al 70\% (EHA) y glicólicos al 10 y $20 \%$ (EG10 y EG20) de la corteza a partir de un polvo moderadamente grueso con pérdida por desecación del 13,53\%, cenizas totales del 1,95\%, cenizas

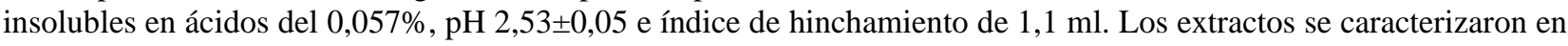
cuanto al contenido de ácido ascórbico y polifenoles totales y la actividad antioxidante determinada por DPPH y ABTS+. El EHA mostró 7011×39 mg/100ml de ácido ascórbico y 7,75\% de polifenoles totales, mientras que en el 
EG10 y EG20 el contenido de ácido ascórbico fue de $3034 \pm 0$ y $6137 \pm 0 \mathrm{mg} / 100 \mathrm{~mL}$ y polifenoles totales de 2,65 y 3,55\%. respectivamente. Los extractos mostraron capacidad antioxidante DPPH con IC50 de 11.80, 48.95 y 25.26 $\mu \mathrm{g} / \mathrm{mL}$ y ABTS+ de $92.91 \pm 0.205,74.59 \pm 0.259$ y 77.21 $11.34 \%$ en los extractos EHA, EG10 y EG20, respectivamente. Los extractos tienen contenidos importantes de compuestos bioactivos, sin embargo, el solvente influyó en el contenido y la actividad biológica, siendo el EHA el de mayor contenido de bioactivos, lo que también se reflejó en su mayor capacidad antioxidante.

Palabras clave: Myrciaria dubia; Antioxidante; Extracto hidroalcohólico; Extracto glicólico.

\section{Introdução}

Os compostos fenólicos, assim como o ácido ascórbico vem sendo conhecidos em razão das propriedades fisiológicas e farmacológicas que conferem à saúde humana atividade antioxidante. Os vegetais, em particular as frutas, apresentam vários compostos com ação antioxidante, os quais incluem o ácido ascórbico, carotenóides e polifenóis (Melo et al., 2008; Bomfim et al., 2017).

O estudo do potencial antioxidante é de grande importância fisiológica, pois muitas doenças e processos degenerativos estão associados à superprodução de radicais livres os quais, têm estimulado grupos de pesquisa a investigarem o potencial antioxidante de extratos de plantas. Na Amazônia, existem várias espécies vegetais que possuem um grande potencial a ser explorado, na forma de alimentos funcionais ou nutracêuticos. Em meio a esta biodiversidade brasileira está a Myrciaria dubia (H.B.K.) McVaugh, conhecida como camu-camu, camocamo, caçari, araçá-d'água, araçá-de-igapó, guayabo e guayabito, uma espécie de porte arbustivo encontrada às margens dos rios, lagos, região de várzea e igapó da Amazônia (Arruda et al., 2011). O fruto de M. dubia, tem despertado interesse no mercado, por apresentar elevado teor de ácido ascórbico, geralmente superior a $2 \mathrm{~g} \times 100 \mathrm{~g}-1$ (Morais \& Pinheiro, 2018) e de outros princípios ativos como antocianinas, com teores médios de $54 \mathrm{mg}$ por $100 \mathrm{~g}$ (Rodrigues \& Marx, 2006; Grigio et al., 2021).

Aponta-se que o fruto de M. dubia, tem grande potencial na melhoria da nutrição e saúde (Castro et al., 2018; Conceição et al., 2020), em função do seu notável conteúdo de polifenóis e vitamina C, maiores que muitas frutas consideradas fontes desses compostos (Yuyama et al., 2002; Zanatta \& Mercadante, 2007; Akter et al., 2011; Santos et al., 2022). A exploração racional desta espécie poderá contribuir para o desenvolvimento sustentável da região por meio do desenvolvimento de bioprodutos (Willerding et al., 2020). Sendo assim, o camu-camu têm potencial para ser usado no desenvolvimento de novos alimentos, medicamentos e cosméticos ricos em bioativos (Fidelis et al., 2020). Desta forma, o objetivo deste estudo, foi avaliar se diferentes extratos obtidos de cascas do fruto de $M$. dubia influencia na atividade antioxidante, já que o fruto vem ganhado visibilidade e o aproveitamento de subprodutos vem se destacando para outras espécies como fonte principalmente de substâncias bioativas.

\section{Metodologia}

\subsection{Material vegetal}

Foram coletados $8 \mathrm{Kg}$ de frutos maduros de Myrciaria dubia (H.B.K.) McVaugh na comunidade de São Domingos, no município de Belterra, no oeste do Estado do Pará ( $2^{\circ} 46^{\prime} 14$ ” S 55¹'42”O), em fevereiro de 2019. Os frutos foram acondicionados em sacolas plásticas e congelados em freezer para uso posterior.

Por se tratar de coleta de um patrimônio da biodiversidade brasileira, foi realizado cadastro no Sistema Nacional de Gestão do Patrimônio Genético e do Conhecimento Tradicional Associado (SisGen), sob número A156833.

\subsection{Obtenção da droga vegetal de Myrciaria dubia}

Frutos de Myrciaria dubia foram previamente descongelados à temperatura ambiente, despolpados manualmente, separando-se a casca, polpa e semente. As diferentes partes do fruto foram pesadas e desidratadas em dessecador de alimentos 
(Excalibur®) a $46^{\circ} \mathrm{C}$ por $24 \mathrm{~h}$. As cascas foram pulverizadas (droga vegetal) foram caracterizadas e usadas para obtenção de extratos hidroalcoólico e glicólicos.

\subsection{Caracterização físico-química da droga vegetal pulverizada}

Os ensaios de caracterização da droga vegetal (granulometria, densidade aparente, pH, índice de intumescência, determinação da perda por dessecação, teor totais de cinzas e cinzas insolúveis em ácido), foram realizados de acordo com o preconizado pela Farmacopéia Brasileira (1999; 2019). Todos os ensaios foram realizados em triplicata.

\subsubsection{Granulometria}

Vinte e cinco gramas da amostra vegetal triturada, foram submetidas à passagem forçada por vibração, através de tamises, com abertura de malhas correspondentes a 600, 425, 250, 150, 125 e $75 \mu \mathrm{m}$, os quais foram previamente pesados. Utilizou-se um tamisador Lab1000® com frequência de vibração 7, durante 15 minutos. Após este processo, foram quantificadas quanto às suas proporções calculando o percentual retido em cada tamis, utilizando o seguinte cálculo:

$\%$ Retirada pelo tamis $=\mathrm{P} 1 / \mathrm{P} 2.100$

$\mathrm{P} 1=$ Peso inicial

$\mathrm{P} 2$ = Peso Final

e posteriormente classificados de acordo com a Farmacopéia Brasileira, $5^{\text {a }}$ Edição (2010).

\subsubsection{Determinação da densidade aparente não compactada}

Em uma proveta de $250 \mathrm{~mL}$ previamente pesada foi adicionada $30 \mathrm{~g}$ de droga vegetal. A densidade aparente foi determinada com os dados de volume e massa, de acordo com os seguintes cálculos:

$$
\mathrm{Dap}=\mathrm{Mpc}-\mathrm{Mpv} / \mathrm{Vp}
$$

Em que:

Dap = densidade aparente;

$\mathrm{Mpv}=$ massa da proveta vazia;

$\mathrm{Mpc}=$ massa da proveta cheia;

$\mathrm{Vp}=$ volume da proveta.

\subsubsection{Determinação do $\mathbf{p H}$}

Foi preparada uma solução aquosa a $1 \%$ com droga vegetal (p/V) por infusão por 15 minutos. Após o resfriamento a mistura foi filtrada e o pH determinado em pHmetro (MS Tecnopon ${ }^{\circledR}$ ).

\subsubsection{Determinação do índice de intumescência}

Foi utilizado $1 \mathrm{~g}$ da droga vegetal em provetas de $25 \mathrm{~mL}$, com adição de água ultrapura mantidos sob agitação a cada 10 minutos por uma hora. As amostras foram deixadas em repouso por 3 horas à temperatura ambiente. Posteriormente, foram verificados os volumes finais ocupados pelas amostras, onde o Índice de intumescência (Ii) foi calculado em mililitros, pela média de 3 determinações segundo a equação abaixo:

$$
\mathrm{Ii}=\mathrm{Vf}-\mathrm{Vi}
$$

Onde, Vf corresponde ao volume final ocupado pela amostra $(\mathrm{mL})$; Vi é o volume inicial ocupado pela amostra (mL). 


\subsubsection{Determinação da perda por dessecação}

Cerca de $0,5 \mathrm{~g}$ da droga vegetal foram colocados em pesa-filtros, previamente tarados. O pesa-filtro foi posto em estufa à $105^{\circ} \mathrm{C} \pm 2^{\circ} \mathrm{C}$, retirando-se a tampa, deixando-a também na estufa, por um período de 2 horas. O pesa-filtro foi esfriado em dessecador por 20 minutos e depois pesado. Este procedimento se repetiu até peso constante. Em seguida, foi calculada a diferença de peso entre a amostra inicial e a final determinando-se a perda por dessecação (Brasil, 2019).

\subsubsection{Determinação do teor de cinzas totais}

Em mufla a $550^{\circ} \mathrm{C}$ por 30 minutos, cadinhos foram calcinados, resfriados em dessecador (15 minutos) e suas massas (taras) foram determinados em balança analítica. Em cada cadinho foram adicionados cerca de $3 \mathrm{~g}$ da droga vegetal triturada, pesados em balança analítica, os quais foram incinerados (levados ao estado de carvão) e, posteriormente, submetidos à calcinação em mufla à temperatura de $550^{\circ} \mathrm{C}$ por seis horas.

Foram deixados em dessecador para arrefecimento (15 minutos), com pesagem realizada posteriormente. A técnica foi repetida até massa constante. $\mathrm{O}$ resultado foi expresso em porcentagem em massa de cinza na droga $(\%, \mathrm{~m} / \mathrm{m})$ e representa a média de três determinações (Brasil, 2019).

\subsubsection{Determinação de cinzas insolúveis em ácido}

Foi fervido o resíduo obtido na determinação de cinzas totais durante 5 minutos com $25 \mathrm{~mL}$ de ácido clorídrico a $7 \%$ (p/v) em cadinho coberto com vidro de relógio. O vidro de relógio, foi lavado com $5 \mathrm{~mL}$ de água quente. Recolheu-se o resíduo, insolúvel em ácido, sobre papel de filtro, isento de cinza, lavando-se com água quente até que o filtrado se mostra-se neutro. O papel de filtro contendo o resíduo para o cadinho original, secou sobre chapa quente e incinerado a cerca de $500^{\circ} \mathrm{C}$ até peso constante. Calculou-se a porcentagem de cinzas insolúveis em ácido em relação à droga seca ao ar (Brasil, 2019).

\subsection{Obtenção de extratos hidroalcoólicos e glicólicos}

O extrato hidroalcoólico (EHA), foi obtido por maceração da droga vegetal em uma proporção de 1:2 (p/v) em etanol/água (70:30, v/v), por um período de 7 dias a temperatura ambiente $\left(20-25^{\circ} \mathrm{C}\right)$ e abrigo de luz. A mistura foi agitada ocasionalmente, filtrada e concentrada em rota evaporador, seguida de estufa a $45{ }^{\circ} \mathrm{C}$ por 24 horas. $\mathrm{O}$ extrato obtido foi acondicionado sob refrigeração e ao abrigo de luz.

Foram preparados extratos glicólicos 10\% (EG10) e 20\% (EG20), submetidos a maceração com solução glicólica (glicerina + álcool de cereais na proporção de 10 ou 20\%) por 7 dias com agitação ocasional e ao abrigo de luz. A mistura foi aquecida a $40^{\circ} \mathrm{C}$ por uma hora seguida de filtração simples para separação do resíduo.

\subsection{Prospecção fitoquímica dos extratos}

Os ensaios de prospecção fitoquímica dos extratos, foram realizados conforme Costa, (2001). Nos extratos foram avaliados os grupos de heterosídeos antraquinônicos (reação de Bornträger); heterosídeos flavonóides (reações de cloreto de alumínio); heterosídeos saponínicos (índice de espuma); taninos (reações com gelatina, cloreto férrico, acetato de chumbo), alcalóides (reativos de Mayer), cumarinas (teste da fluorescência, sob luz UV).

\subsection{Determinação de ácido ascórbico pelo método de Tillmans}

O teor de ácido ascórbico dos extratos foi realizado pelo método de Tillmans, segundo as normas do Instituto Adolf Lutz, (2008).

Para obtenção da solução padrão, pipetou-se $10 \mathrm{~mL}$ da solução padrão de ácido ascórbico em erlenmeyer contendo 50 
mL de solução de ácido oxálico. Titulou-se com solução de 2,6-diclorofenolindofenol sódio até coloração rosa persistente por 15 segundos. O volume gasto na titulação foi dividido por 5. O resultado é o volume em mL gastos para titulação de $1 \mathrm{mg}$ de ácido ascórbico.

Foi adicionado a amostra em $50 \mathrm{~mL}$ da solução de ácido oxálico no erlenmeyer e titulou-se com a solução de 2,6diclorofenolindofenol sódio padronizado, até coloração rosa persistente por 15 segundos. O resultado foi expresso em mg de ácido ascórbico por $100 \mathrm{~mL}$ ou $100 \mathrm{~g}$ da amostra, pela fórmula.

$$
\mathrm{AA}=\frac{100 \times \mathrm{n}^{\prime}}{\mathrm{n} / 5 \times \mathrm{P}}
$$

Onde:

$\mathrm{AA}=$ Teor de ácido ascórbico em mg/100 mL ou mg/100 g.

n' = Volume de 2,6-diclorofenolindofenol sódio em mL gastos na titulação da amostra.

$\mathrm{n}=$ Volume de 2,6-diclorofenolindofenol sódio em $\mathrm{mL}$ gastos na padronização.

$\mathrm{P}=$ Massa da amostra em grama ou volume de amostra usado na titulação.

\subsection{Determinação de polifenóis totais}

Os teores de polifenóis totais presentes nos extratos foram determinados pelo método de Hagerman e Butter (Mole \& Walterman, 1987) com modificações.

Alíquotas de $100 \mu \mathrm{L}$ dos extratos foram diluídas em $10 \mathrm{~mL}$ de metanol a 20\% (v/v). Em tubos de ensaio contendo 2 $\mathrm{mL}$ de solução aquosa de $\mathrm{LSS} /$ trietanolamina/ isopropanol, $1 \mathrm{~mL}$ de solução de $\mathrm{FeCl}_{3}$, foi adicionado alíquotas de $1 \mathrm{~mL}$ das soluções dos extratos diluídas. As amostras ficaram em repouso por 15 minutos e as absorbâncias lidas em $510 \mathrm{~nm}$. O branco foi preparado substituindo a amostra por água destilada.

Para a construção da curva de calibração foram pesados $25 \mathrm{mg}$ de ácido tânico e transferidos para balão volumétrico de $25 \mathrm{~mL}$, completando-se o volume com água destilada. Alíquotas de 100, 200, 300, 400, 500,600 e $800 \mu \mathrm{L}$ desta solução foram transferidas para tubos de ensaio contendo a solução de LSS/ trietanolamina/ isopropanol e a solução de $\mathrm{FeCl}_{3}$. $\mathrm{O}$ valor da absorbância foi substituído na variável y, da equação y=a+bx, obtendo-se a concentração (variável x) em mg.mL-1.

Para calcular a porcentagem de fenóis totais presentes nas amostras utilizou-se as seguintes fórmulas:

$$
\mathrm{c}=\text { Absorbância }-\mathrm{A} / \mathrm{B}
$$

$\%$ Fenóis totais $=\mathrm{c} \times 250 \times 10-3 \times 100 / \mathrm{m}(\mathrm{g})$

onde:

$\mathrm{c}=$ concentração de ácido tânico em $\mathrm{mg} / \mathrm{mL}$

$\mathrm{A}=$ coeficiente linear da equação da reta

$\mathrm{B}=$ coeficiente angular da equação da reta

$\mathrm{m}=$ massa em gramas

\subsection{Avaliação da atividade antioxidante dos extratos por DPPH}

A atividade sequestradora do radical livre DPPH, dos extratos glicólicos e hidroalcoólicos foram obtidas conforme descrito por Brand-Williams et al., (1995), com modificações.

Para a análise das amostras, adicionou-se a 1,5 mL da solução metanólica de DPPH (6x10-5M) uma alíquota de 0,5 $\mathrm{mL}$ das amostras contendo diferentes concentrações de cada extrato. As leituras foram realizadas em espectrofotômetro 
(Spectrophotometer Model nova 3300 UV) a 517 nm, após 5; 10; 15; 30; 45 e 60 minutos do início da reação. Todas as determinações foram realizadas em triplicata e acompanhadas de um controle (sem antioxidante). A queda na leitura da absorbância das amostras foi correlacionada com o controle (água), estabelecendo-se a porcentagem de descoloração do radical DPPH, conforme a fórmula abaixo:

$$
\% \text { proteção }=[(\text { Abscontrole }- \text { Absbranco }) / \text { Abscontrole }] \times 100
$$

Para o cálculo dos valores de EC50 (concentração do extrato necessário para reduzir $50 \%$ do radical DPPH), foi calculada a atividade antioxidante em diferentes concentrações, de forma a traçar uma curva linear entre a capacidade antioxidante do respectivo extrato e sua concentração. Esses dados foram submetidos a uma regressão linear e obtida uma equação da reta para o cálculo do EC50.

AAO (\%inibição $)=100-($ A amostra - A branco $)$ x 100

\subsection{Determinação da atividade antioxidante dos extratos pelo método de $\mathrm{ABTS}^{+}$}

A atividade antioxidante dos extratos, através da captura do cátion radical com o ácido 2,2-azino-bis(3etilbenzotiazolin)-6-sulfônico - $\mathrm{ABTS}^{+}$, foi determinada conforme descrição de metodologia (Rufino et al, 2007). Foi utilizado o trolox como padrão antioxidante e os resultados foram expressos em termos da capacidade antioxidante do composto equivalente ao trolox, expresso em valor de TEAC (Trolox Equivalent Antioxidant Capacity, capacidade antioxidante total do composto equivalente ao trolox). A solução do cátion radical foi preparada pela reação do $\mathrm{ABTS}^{+} 3,5 \mathrm{mM}$ com persulfato de potássio $140 \mathrm{mM}$.

Para completa reação e estabilização do radical, a solução radical $\mathrm{ABTS}^{+}$permaneceu ao abrigo de luz, à temperatura ambiente, por um período de 16 horas. Foi diluído a solução de $\mathrm{ABTS}^{+}$em etanol até obter uma absorbância de 0,7 $\pm 0,05$ a $734 \mathrm{~nm}$. A curva de calibração do padrão trolox foi feita nas concentrações de 100, 250, 500 e $1000 \mu \mathrm{M}$. As concentrações utilizadas para construção da curva de calibração para capacidade antioxidante dos extratos foram de 1000, 2000, 4000, 6000, 8000 e $10000 \mu \mathrm{g} / \mathrm{mL}$. Em ambiente escuro foi transferida uma alíquota de $30 \mu \mathrm{L}$ de cada solução padrão, para tubos de ensaio e adicionados 3,0 mL da solução de radical $\mathrm{ABTS}^{+}$. As absorbâncias foram medidas a $734 \mathrm{~nm}$ após 6 min de reação, utilizando-se o etanol como branco. Foi realizado o mesmo procedimento para as soluções de extratos. A atividade em capturar o cátion radical $\mathrm{ABTS}^{+}$foi expressa em $\mu \mathrm{M}$ trolox/g de OEMS (Z) e obtida a partir das equações das retas das curvas: concentração de trolox versus absorbância e concentração da amostra versus absorbância. A absorbância foi medida em espectrofotômetro, no tempo de 6 minutos após adição da amostra. Os experimentos foram realizados em triplicata (RiceEvans et al., 1996).

\subsection{Análise estatística}

Os dados obtidos para os experimentos realizados representam a média seguida do erro padrão da média (EPM) e foram comparados por análise de variância pelo teste ANOVA, seguido do teste de Tukey $(p<0,05)$.

\section{Resultados e Discussão}

\subsection{Rendimento da droga vegetal}

Quando o material vegetal é submetido à secagem, ocorre uma redução considerável da sua massa seca em relação à massa fresca. O peso inicial da casca foi de $2962 \mathrm{~g}$ e após o processamento esse valor reduziu para $332 \mathrm{~g}$. O rendimento percentual da casca desidratada e pulverizada de Myrciaria dubia foi de 11,20\%. 


\subsection{Granulometria}

Na Tabela 1 estão os dados da caracterização granulométrica da droga vegetal. O pó obtido pela pulverização da casca desidratada de Myrciaria dubia foi classificado conforme a Farmacopéia Brasileira $5^{\text {a }}$ ed. (2010), como pó moderadamente grosso, no que se refere às partículas que passam em sua totalidade pelo tamis de malha $710 \mu$ m e no máximo $40 \%$ pelo tamis de malha $250 \mu \mathrm{m}$. O processo de pulverização da droga é extremamente importante, pois a mesma auxilia no processo de extração de drogas vegetais. Zeng et al., (2007) e Liu et al., (2013), afirmam que reduzir o tamanho das partículas, torna a sua superfície de contato maior, melhorando a transferência de calor e massa no processo. Além disso, a moagem provoca alterações na estrutura que podem reduzir a cristalinidade das fibras de celulose. De acordo com Sharapin (2000), os pós mais grossos são os mais recomendados para a maioria das drogas vegetais, visto que os pós finos podem comprometer o processo de filtração, dificultando a passagem do solvente e ocasionado uma extração incompleta. Desta forma, os pós grossos ou moderadamente grossos são os mais recomendados para o processo de extração.

Tabela 1 - Análise granulométrica de casca pulverizada de Myrciaria dubia (H.B.K.) McVaugh.

\begin{tabular}{lcc}
\hline \multicolumn{1}{c}{ Tamis/Tamanho de partículas retidas } & Orifício do Tamis $(\boldsymbol{\mu m})$ & Casca $(\%)$ \\
\hline ASTM USS 30 & 600 & 31,61 \\
Partículas maiores que $600 \mu \mathrm{m}$ & 425 & 20,38 \\
ASTM USS 40 & & 28,10 \\
Partículas entre 600 e $425 \mu \mathrm{m}$ & 250 & 11,44 \\
ASTM USS 60 & 180 & 1,272 \\
Partículas entre 425 e $250 \mu \mathrm{m}$ & & 3,80 \\
ASTM USS 80 & 125 & 3,40 \\
Partículas entre 250 e $180 \mu \mathrm{m}$ & 75 & \\
Partículas entre 180 e $125 \mu \mathrm{m}$ & & \\
ASTM USS 200 & - & \\
Partículas entre 125 e $75 \mu \mathrm{m}$ & & \\
Coletor Partículas menores que $75 \mu \mathrm{m}$ & & \\
\hline
\end{tabular}

Fonte: Autores.

\subsection{Perda por dessecação, Cinzas Totais, Cinza Insolúveis em ácido, pH, densidade aparente não compactada e Índice de intumescência}

Na Tabela 2 estão os dados de umidade, cinzas totais, cinzas insolúveis em ácido, pH e densidade aparente não compactada e índice de intumescência. Os valores obtidos para a perda por dessecação da casca pulverizada de Myrciaria

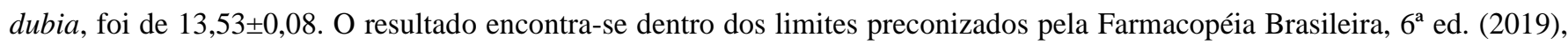
para drogas vegetais, que variam de 8 a $14 \%$ de umidade para a droga seca. Este resultado assegura a estabilidade microbiológica e química da droga vegetal, visto que, os teores de umidade elevados acima do determinado, levam a proliferação de bactérias e fungos, e possíveis degradações de substâncias químicas por processos de hidrólise mesmo à curto prazo (Schimidt, 1989). Sendo assim, o resultado está dentro dos parâmetros estabelecidos para minimizar a contaminação microbiana e degradação química.

A determinação do teor de cinzas totais permitiu verificar a presença de substancias inorgânicas não voláteis presente na droga vegetal. Quanto mais baixo forem os resultados obtidos para teor de cinzas totais, menor será a quantidade de compostos inorgânicos não voláteis presentes na amostra (Barni et al., 2009; Brasil, 2010). Conforme os resultados obtidos na determinação de cinzas totais pode-se verificar que a amostra analisada possui uma quantidade muito baixa de matéria inorgânica (1,95\%). Elevados teores de cinzas pode ser indício de contaminação elevada por areia e/ou terra, indicando um mau tratamento na colheita e/ou beneficiamento das plantas frescas (Guizzo, et al., 2015). O conteúdo das cinzas insolúveis em 
ácido clorídrico das amostras foi, em média, 0,05\%. De acordo com Park (1996), altos níveis de cinzas insolúveis em ácido indica presença de areia ou sujeira nas amostras. O método destina-se à determinação de sílica e constituintes silícicos da droga. (Brasil, 2019).

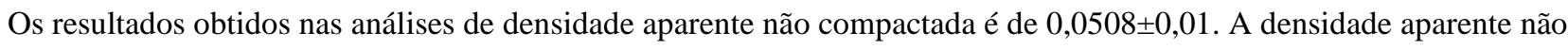
compactada é utilizada na especificação de embalagens, transporte e armazenamento. Um grande número de espaços vazios presentes entre as partículas do pó explica uma menor densidade. Esta depende de uma série de fatores como: densidade de seus componentes, geometria e tamanho da partícula, sua distribuição granulométrica e o método de análise (Lewis, 1993).

$\mathrm{O}$ valor de $\mathrm{pH}$ da droga vegetal analisada $(2,53 \pm 0,05)$. Esses resultados determinam a caracterização da matériaprima, servindo como mais um parâmetro de qualidade (Brasil, 2019).

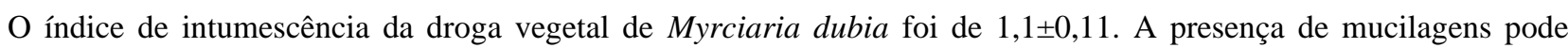
indicar atividades emolientes, e permitem a produção de xaropes com propriedades expectorantes e demulcentes (Hoelzel, 2003). Ademais, trata-se de um parâmetro para processos extrativos, auxiliando na quantidade de água a ser adicionado durante a extração de substâncias pécticas (Who, 2011).

Tabela 2 - Perda por dessecação, Cinzas Totais, Cinza Insolúveis em ácido, pH, Densidade aparente não compactada e Índice de intumescência de casca pulverizada de Myrciaria dubia (H.B.K.) McVaugh.

\begin{tabular}{cc}
\hline Ensaio & Resultado (\%) (Média \pm desv.pad) \\
\hline Perda por dessecação & $13,53 \pm 0,08$ \\
Cinzas Totais & $1,95 \pm 0,03$ \\
Cinzas insolúveis em ácidos & $0,057 \pm 0,00$ \\
pH & $2,53 \pm 0,05$ \\
Densidade aparente não compactada & $0,0508 \pm 0,01$ \\
Índice de intumescência & $1,1 \pm 0,11$ \\
\hline
\end{tabular}

Os resultados representam a média \pm erro padrão da média $(\mathrm{n}=3)$. Fonte: Autores.

\subsection{Rendimento dos extratos de Myrciaria dubia}

Foram obtidos 3 extratos de casca pulverizada de Myrciaria dubia, sendo dois extratos glicólicos 10 (EG10) e 20\% (EG20) e um extrato hidroalcoólico (EHA). Os dados de rendimento dos extratos estão apresentados na Tabela 3. Houve uma redução no rendimento de EG20 quando comparado ao EG10. A quantidade de droga vegetal influenciou na fluidez do extrato o que pode ter prejudicado o seu rendimento.

Os procedimentos de extração são um passo importante no processamento dos constituintes bioativos derivados de materiais vegetais (Azwanida, 2015). O tipo de solvente utilizado e as técnicas extrativas influenciam diretamente no cálculo de rendimento (Oliveira et al., 2016). O sistema hidroalcoólico é bastante eficaz na extração de diversos componentes químicos presentes em plantas, haja vista, sua capacidade de penetrar na membrana celular para extrair os ingredientes intracelulares do material botânico (Tiwari et al., 2011).

Tabela 3 - Extrato e rendimento em (\%) de casca pulverizada de Myrciaria dubia (H.B.K.) McVaugh.

\begin{tabular}{cc}
\hline Extratos & Rendimento (\%) \\
\hline EHA & $70,66^{\mathrm{a}}$ \\
EG10 & $58^{\mathrm{b}}$ \\
EG20 & $34^{\mathrm{c}}$ \\
\hline
\end{tabular}

Os resultados representam a média \pm erro padrão da média $(\mathrm{n}=3)$. Letras diferentes na mesma coluna indicam diferença significativa $(\mathrm{p}<0,05)$ pelo teste de Tukey. Fonte: Autores.

\subsection{Prospecção fitoquímica dos extratos de Myrciaria dubia}

Na tabela 4 estão os dados preliminares de prospecção fotoquímica dos extratos hidroalcoólico e glicólicos. Esses 
achados estão relacionados ao expresso por Herrera-Calderón et al., (2018) ao relatar que extratos etanólicos de Myrciaria dubia contém alcalóides, flavonóides, fenóis, terpenos e esteróides.

Observa-se que ambos os extratos apresentaram saponinas e taninos. A saponina foi caracterizada pela formação de espuma persistente e abundante. Devido seu caráter anfifílico, conhecidas por suas propriedades emulsificantes e detergentes em diversos setores industriais (Tanaka et al., 1996; Mitra \& Dungan, 1997).

Os taninos foram identificados pela precipitação e mudança de cor. Pelo teste de cloreto de ferro, observou-se que o extrato EHA apresentou coloração verde e os extratos EG10 e EG20, apresentaram coloração azul, lhe caracterizando como taninos condensados e gálicos respectivamente. Na mesma planta podem ocorrer, simultaneamente, taninos hidrolisáveis e condensados predominando um destes tipos. Os taninos condensados e hidrolisados, possuem atividade antioxidante e sequestradora de radicais livres e capacidade de complexar macromoléculas de natureza proteica e íons metálicos, além de intervirem na modulação de processos envolvidos na divisão e proliferação celular, na coagulação, inflamação e resposta imunológica (Jerez et al., 2006).

Tabela 3 - Triagem fitoquímica de extratos glicólicos e hidroalcoólicos de Myrciaria dubia (H.B.K.) McVaugh.

\begin{tabular}{ccccc}
\hline Metabólito Secundário & Ensaio & EHA & EG10 & EG20 \\
\hline Antraquinonas & Reação de Bornträger & - & - & - \\
Heterosídeos flavonóides & reações com cloreto de alumínio & - & - & - \\
Saponina & Teste qualitativo de espuma & + & + & + \\
Taninos & Cloreto Férrico & TC & TG & TG \\
Alcalóides & Acetato de chumbo & - & - & - \\
Cumarinas & Reativos de Mayer & - & - & - \\
\hline
\end{tabular}

Legenda: EHA= Extrato Hidroalcoólico; EG10= Extrato Glicólico 10\%; EG20 = Extrato Glicólico 20\%; TC = Tanino Condensado; TG = Tanino Gálico. Fonte: Autores.

\subsection{Determinação de ácido ascórbico pelo método de Tillmans}

$\mathrm{Na}$ Tabela 4 estão os dados do teor de vitamina $\mathrm{C}$, por titulometria. $\mathrm{O}$ teor de vitamina $\mathrm{C}$ foi maior para o extrato EHA. No entanto, observa-se que o EG20 foi duas vezes maior que o extrato EG10.

Os frutos de camu-camu procedentes do estado de Roraima e Amazônia central, em geral mostram teor de vitamina C que varia amplamente entre 0,934 a 6,112 g/100g de polpa (Andrade et al., 1991.; Yuyama et al., 2002). A ampla variação do teor de vitamina $\mathrm{C}$ entre as diferentes populações se deve em grande parte as diferenças genéticas e as técnicas de extração (Teixeira et al., 2004). Locais de coleta também influenciam nesses valores, no estudo de Abanto-Rodriguez et al., (2016), o teor de vitamina $C$ teve variação entre as três zonas de produção de camu-camu no Ucayali, Peru, com valores de 2.489,25, 2134,5 e $2088,6 \mathrm{~mm} / 100 \mathrm{~g}$ de polpa.

Tabela 4 - Teores de ácido ascórbico de diferentes extratos de Myrciaria dubia (H.B.K.) McVaugh.

\begin{tabular}{cc}
\hline Extrato & Valor $(\mathbf{m g} / \mathbf{1 0 0 m l})$ \\
\hline EHA & $7011 \pm 39^{\mathrm{a}}$ \\
EG10 & $3034 \pm 0^{\mathrm{b}}$ \\
EG20 & $6137 \pm 0^{\mathrm{a}}$ \\
\hline
\end{tabular}

EHA $=$ Extrato Hidroalcoólico; EG10 = Extrato Glicólico 10\%; EG20 = Extrato Glicólico 20\%. Os resultados representam a média \pm erro padrão da média $(n=3)$. Letras diferentes na mesma coluna indicam diferença significativa $(\mathrm{p}<0,05)$ pelo teste de Tukey. $*$ Os resultados representam a média \pm erro padrão da média $(\mathrm{n}=3)$. Fonte: Autores.

\subsection{Determinação de polifenóis totais}

Para a quantificação de compostos fenólicos totais, foi necessária a construção de uma curva padrão de ácido tânico, 
conforme está representado na Figura 1.

Figura 1. Curva padrão para o doseamento de fenóis totais em Myrciaria dubia. Concentração de ácido tânico em $\mathrm{mg} / \mathrm{mL}$ versus Absorbância (ABS). Cada ponto representa a média \pm desvio padrão em triplicata.

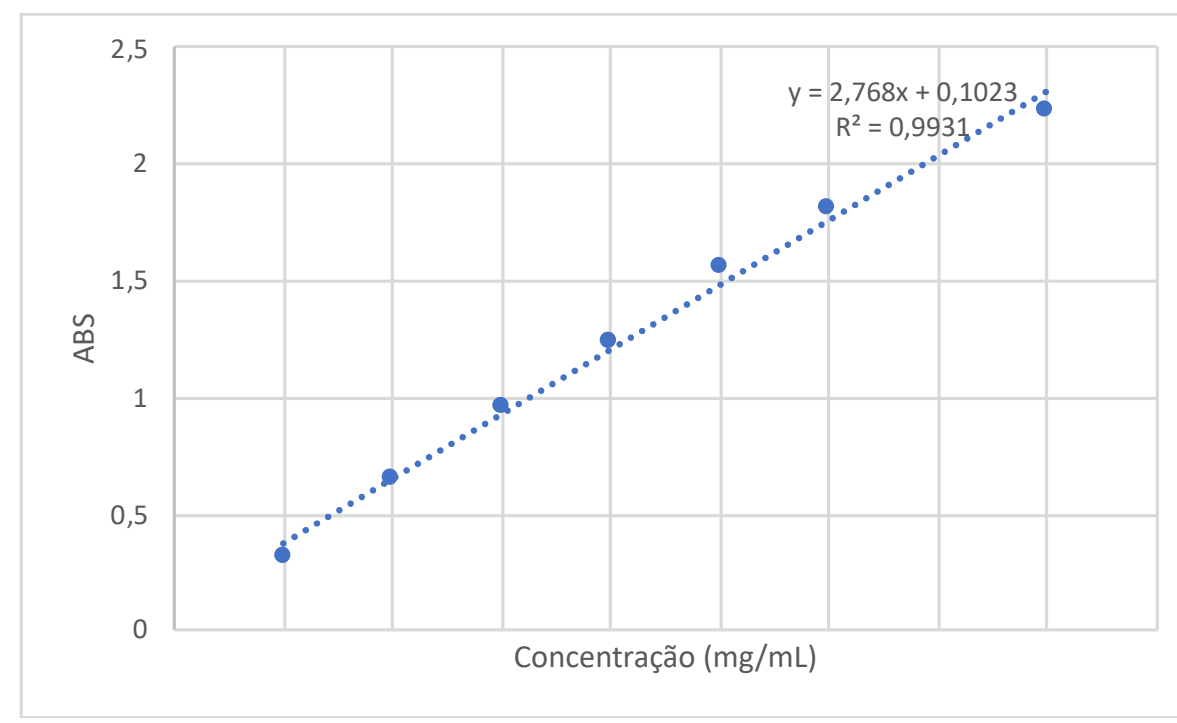

Fonte: Autores.

Como observado na Tabela 5, a fração fenólica do extrato EHA (7,75\%) foi aproximadamente duas vezes maior que EG10 (2,61\%) e EG20 (3,55\%). O teor de compostos fenólicos presentes em uma amostra tem correlação linear com a capacidade antioxidante, bem como o método extrativo (Ignat et al., 2011). Segundo Macheix et al., (1990), compostos fenólicos são encontrados em maior quantidade no epicarpo (casca) de frutas. Dentre as frutas amazônicas, a Myrciaria dubia se destaca não só pelo alto teor de vitamina C (Zapata \& Dufour, 1993; Maeda et al., 2006; Rufino et al., 2010), mas também por possuir uma quantidade significativa de compostos fenólicos.

Tabela 5 - Porcentagem (m/m) de compostos fenólicos totais (FT) para os extratos de cascas dos frutos de Myrciaria dúbia.

\begin{tabular}{ll}
\hline Amostra & FT (\%) (Média \pm desv.pad) \\
\hline EHA & $7,75 \pm 0,031^{\mathrm{a}}$ \\
EG10 & $2,61 \pm 0,0005^{\mathrm{b}}$ \\
EG20 & $3,55 \pm 0,01^{\mathrm{b}}$ \\
\hline
\end{tabular}

Legenda: EHA = Extrato Hidroalcoólico; EG10 = Extrato Glicólico 10\%; EG20= Extrato Glicólico 20\%. Os resultados representam a média \pm erro padrão da média $(\mathrm{n}=3)$. Letras diferentes na mesma coluna indicam diferença signifi cativa $(\mathrm{p}<0,05)$ pelo teste de Tukey. Fonte: Autores.

Segundo Neves at al., (2015), a concentração de fenóis totais nos frutos de camu-camu variam entre tempos de colheita com concentrações médias de 12798,80 e 14854,00 mg GAE / amostra de 100 g, os quais estavam contidos na polpa e na casca, com níveis maiores de concentração em estágios verde-avermelhas. Este resultado corrobora com os dados de Chirinos et al., (2010) e Villanueva-Tiburcio et al., (2010), que observou alta concentrações de fenol em frutos de camu-camu durante vários estágios de maturação com base na cor da casca e concentração diminuída entre a maturação intermediária estágio e o estágio totalmente maduro. Alves et al., (2002), observou uma concentração mais baixa de fenóis totais em frutas completamente roxas do que em frutos totalmente verdes para todas as soluções de extração. 


\subsection{Avaliação da atividade antioxidante dos extratos por DPPH e ABTS ${ }^{+}$}

O radical DPPH é útil para avaliar a atividade antirradical de polifenóis bem como a toxicidade em células e extratos microbianos (Sánchez-Moreno; et al., 1998; Lebeau et al., 2000). Os resultados da atividade antioxidante por DPPH e ABTS ${ }^{+}$ dos extratos de casca de Myrciaria dubia estão na tabela 6.

Todos os extratos apresentaram atividade antioxidante frente ao DPPH, no entanto, o extrato EHA (88\%) e EG20 (54\%), foram mais efetivos. Indicando o grande potencial funcional e capacidade antioxidante dos frutos de camu-camu, com ótimo potencial para aplicação e desenvolvimento como produto funcional (Fracassetti et al., 2013; Fujita et al., 2013; Neves et al., 2015; Solis et al., 2009; Rufino et al., 2010; Villanueva-Tiburcio et al., 2010).

Estudos de Neves et al., (2015), Chirinos et al., (2010), mostram que os resultados da atividade antioxidante (determinada por DPPH) é influenciado pelo alto poder redutor do ácido ascórbico, presente em concentrações variadas durante diferentes camu-camu estágios de maturação. Da mesma forma, que a capacidades antioxidantes da fruta seguiu a mesma tendência que a concentração total de fenol.

O resultado obtido para a capacidade antioxidante pelo método de $\mathrm{ABTS}^{+}$, foi de $92,91 \mu \mathrm{M}$ trolox / $\mathrm{g} \pm$ 0,205 para EHA, 74,59 $\mathrm{mg} \mu \mathrm{M}$ trolox / $\mathrm{g} \pm$ 0,259 para EG10 e 77,21 $\mu \mathrm{M}$ trolox / $\mathrm{g} \pm 1,34$ para EG20.

Frutos frescos de camu-camu tem maior capacidade antioxidante (método do ABTS ${ }^{+}$), Rufino et al. (2010) em seu estudo com 18 frutas brasileiras analisadas, constatou que o camu-camu fresco possui 153 $\pm 2,6 \mu$ mol Trolox/g fruta fresca. Já em ensaios de capacidade antioxidante de $10 \%$ de maltodextrina em pó pulverizado de camu-camu, realizado por Fracassetti et al., (2013), relatou 167,5 $\mu \mathrm{Mol}$ de trolox equivalente /100 g de pó de acordo com o ensaio $\mathrm{ABTS}^{+}$, mostrando que o pó de camu-camu é uma boa fonte de compostos potencialmente bioativos benéficos para a saúde. Os valores encontrados no trabalho, comparados com a literatura, sugerem que a conservação do fruto e tipo de extração refletem na sua capacidade antioxidante.

Tabela 6 - Atividade antioxidante DPPH, Porcentagem de inibição e IC50 e ABTS ${ }^{+}$.

\begin{tabular}{|c|c|c|c|}
\hline \multirow[t]{2}{*}{ Extrato } & \multicolumn{2}{|c|}{ DPPH } & \multirow{2}{*}{$\begin{array}{c}\text { ABTS }^{+} \\
(\boldsymbol{\mu M} \text { trolox } / \mathbf{g})\end{array}$} \\
\hline & \%Inibição & $(\mathrm{IC50})(\mu \mathrm{g} / \mathrm{mL})$ & \\
\hline EHA & $88,027 \pm 0,439^{a}$ & $11,80^{\mathrm{a}}$ & $92,91 \pm 0,205^{\mathrm{a}}$ \\
\hline EG10 & $32,136 \pm 2,312^{b}$ & $48,95^{\mathrm{b}}$ & $74,59 \pm 0,259^{b}$ \\
\hline EG20 & $54,405 \pm 3,282^{\mathrm{c}}$ & $25,26^{\mathrm{c}}$ & $77,21 \pm 1,34^{\mathrm{b}}$ \\
\hline
\end{tabular}

Legenda: EHA = Extrato Hidroalcoólico; EG10 = Extrato Glicólico 10\%; EG20= Extrato Glicólico 20\%. Os resultados representam a média \pm erro padrão da média $(\mathrm{n}=3)$. Letras diferentes na mesma coluna indicam diferença significativa $(\mathrm{p}<0,05)$ pelo teste de Tukey. Fonte: Autores.

\section{Conclusão}

Os extratos hidroalcoólicos e glicólicos obtidos de casca do fruto de Myrciaria dubia, apresentam capacidade antioxidante, isso devido ao alto teor de compostos bioativos como polifenóis e ácido ascórbico. O extrato hidroalcoólico, apresentou o maior conteúdo de compostos bioativos o que refletiu também na sua maior capacidade antioxidante.

Ambos os extratos obtidos de casca de M. dubia poderão ser usados em diferentes formulações para elaboração de bioprodutos principalmente em cosméticos como substitutos de antioxidantes sintéticos.

\section{Referências}

Abanto-Rodriguez, C., et al. (2016). Relação entre os nutrientes minerais e o teor de vitamina C em plantas camu-camu (Myrciria dubia) cultivadas em solos altos e solos de inundação de Ucayali, Peru. Scientia Agropecuaria. Trujillo, 7 (3), 297-304. 10.17268/sci.agropecu.2016.03.18

Akter, M. S., et al. (2011). Nutritional compositions and health promoting phytochemicals of camu-camu (Myrciaria dubia) fruit: A review. Food Research International, 44 (7), 1728-1732. 10.1016/j.foodres.2011.03.045 
Alves, R. E., et al. (2002). CamuCamu ([Myrciaria dubia (H.B.K.) Mc Vaugh] Mc Vaugh): a rich natural source of vitamin C. Proc. Interam. Soc. Trop. Hortic, 46, 11-13.

Andrade, J. S., et al. (1991). Valor nutricional do camu-camu (Myrciaria dubia (H.B.K.) Mc Vaugh Myrtcaceae) cultivado em terra firme na Amazônia Central. Revista Brasileira de Fruticultura, 13 (3), 307-311.

Arruda A. S., et al. (2011). Desenvolvimento do camu-camu (Myrciaria dubia) em diferentes substratos nas condições de Ipameri-GO, Enciclopédia Biosfera, 7, 01-07.

Azwanida, N. N. (2015). A Review on the Extraction Methods Use in Medicinal Plants, Principle, Strength and Limitation. Medicinal \& Aromatic Plants, 4 (3), 01-06. 10.4172/2167-0412.1000196

Barni, S. T. \& Cechinel Filho, V. \& Couto, A. G. (2009). Caracterização química e tecnológica das folhas, caules e planta inteira da Ipomoea pes-caprae (L.) R. Br., Convolvulaceae, como matéria-prima farmacêutica. Revista Brasileira de Farmacognosia, 19, 865-870. 10.1590/S0102-695X2009000600012

Bomfim, M. P., et al. (2017). Caracterização dos compostos bioativos em frutas e hortaliças adquiridas no comércio de Padova-Itália. Revista Iberoamericana de Tecnología Postcosecha, 18 (2), 82-92.

Brand-Williams, W. \& Cuverlier, M. E.\& Berset, C. (1995). User of free radical method to evaluate antionxidant activity. Lebensmittel-Wissenchaft Technologie, 28, 25-30. 10.1016/S0023-6438(95)80008-5

Brasil. (1999). Farmacopéia Brasileira. (4a ed.), Agência Nacional de Vigilância Sanitária.

Brasil. (2010). Farmacopéia Brasileira. (5a ed.), Agência Nacional de Vigilância Sanitária.

Brasil. (2019). Farmacopéia Brasileira. (6a ed.), Agência Nacional de Vigilância Sanitária.

Castro, J. C. \& Maddox, J. D. \& Imán, S. A. (2018). Camu-camu - Myrciaria dubia (Kunth) McVaugh. In. Rodrigues, S.; Silva, E. O. (editores). Exotic Fruits, Academic Press, 97-105. 10.1016/B978-0-12-803138-4.00014-9

Chirinos, R., et al. (2010). Antioxidant compounds and antioxidant capacity of Peruvian camu-camu (Myrciaria dubia (H.B.K.) Mc Vaugh) fruit at different maturity stages. Food Chem. 120, 1019-1024. 10.1016/j.foodchem.2009.11.041

Conceição, N., et al. (2020). By-Products of Camu-Camu [Myrciaria dubia (Kunth) McVaugh] as Promising Sources of Bioactive High Added-Value Food Ingredients: Functionalization of Yogurts. Melecules, 25 (1),1-17. 10.3390/molecules25010070

Costa, A. F. (1972). Farmacognosia. Fundação Colouste Gulbenkian, 2 (3), 1032.

Fidelis, M., et al. (2020). From byproduct to a functional ingredient: Camu-camu (Myrciaria dubia) seed extract as an antioxidant agent in a yogurt model. Journal of dairy science, 103 (2), 1131-1140. 10.3168/jds.2019-17173

Fracassetti, D., et al. (2013). Derivados do ácido elágico, elagitaninos, proantocianidinas e outros fenólicos, vitamina C e capacidade antioxidante de dois produtos em pó da fruta camu-camu (Myrciaria dubia). Química alimentar, 139 (1-4), 578-588.

Fujita A., et al. (2013). Impact of spouted beddrying on bioactive compounds, antimicrobial and antioxidant activities of commercial frozen pulp of camucamu (Myrciaria dubia Mc. Vaugh). Food Res. 54, 495-500. 10.1016/j.foodres.2013.07.025

Góis, F. L. (2021). Controle de qualidade e perfil metabólico de drogas vegetais associadas para o preparo de chás, comercializadas em Salvador-Bahia, Brasil.

Grigio, M. L., et al. (2021). Nutraceutical potential, quality and sensory evaluation of camu-camu pure and mixed jelly. Food Science and Technology. $10.1590 /$ fst.03421

Hagerman, A. E. \& Butler, L.G. (1989). Choosing apropriate methods and standards for assaying tannin. Journal of Chemical Ecology, New York, 15 (6), 1795-1810. 10.1007/BF01012267

Herrera-Calderon, O., et al. (2018). Phytochemical screening, total phenolic content, antioxidant, and cytotoxic activity of five peruvian plants on human tumor cell lines. Pharmacogn. Res. 10, 161-165. 10.4103/pr.pr_109_17

Hoelzel, S. C. S. M., et al. (2003). Estudo Morfo-Anatômico da Raiz de Waltheria douradinha Saint Hilaire (Sterculiaceae). Acta farmaceutica bonaerense, 22 (2), 113-8.

Ignat, I. \& Volf, I. \& Popa, V. I. (2011). A critical review of methods for characterisation of polyphenolic compounds in fruits and vegetables. Food chemistry, 126 (4), 1821-1835. 10.1016/j.foodchem.2010.12.026

Instituto Adolfo Lutz. (2008). Métodos físico-químicos para análises de alimentos. Normas Analíticas do Instituto Adolfo Lutz. 4, 1000.

Jerez, M., et al. (2006). Influence of extraction conditions on phenolic yields from pine bark: Assessment of procyanidins polymerization degree by thiolysis. Food Chemistry, 94 (3), 406-414. 10.1016/j.foodchem.2004.11.036

Lebeau, J., et al. (2000). Antioxidant properties of di-tert-butylhydroxylated flavonoids, Free Radic. Biol. Med., 29 (9), 900-912. 10.1016/S08915849(00)00390-7

Lewis, M. J. (1993). Propiedades físicas de los alimentos y de los sistemas de procesado.Zaragoza: Editorial Acribia. 39-41.

Liu, Z. H., et al. (2013). Effects of biomass particle size on steam explosion pretreatment performance for improving the enzyme digestibility of corn stover. Industrial Crops and Products, 44, 176-184. 10.1016/j.indcrop.2012.11.009 
Research, Society and Development, v. 11, n. 2, e5811225130, 2022

(CC BY 4.0) | ISSN 2525-3409 | DOI: http://dx.doi.org/10.33448/rsd-v11i2.25130

Macheix, J.J. \& Fleuriet, A. \& Billot, J. (1990). Fruit phenolics. Boca Raton, Florida, (ed.) CRC Pres, 378. 10.1080/10408399109527552

Maeda, R. N., et al. (2006). "Determinação da formulação e caracterização do néctar de camu-camu (Myrciaria dubia McVaugh)." Food Science and Technology, 26, 70-74. 10.1590/S0101-20612006000100012

Melo, E. A. \& Guerra, N. B. (2002). Ação antioxidante de compostos fenólicos naturalmente presentes em alimentos. Bol. SBCTA, 36 (1), 1-11.

Melo, E. A., et al. (2008). Capacidade antioxidante de frutas. Revista Brasileira de Ciências Farmacêuticas, 44, 193-201. 10.1590/S151693322008000200005

Mitra, S. \& Dungan, S. R. (1997). Micellar properties of Quillaja saponin. 1. Effects of temperature, salt, and pH on solution properties. Journal of agricultural and food chemistry, 45 (5), 1587-1595.10.1021/jf960349z

Mole, S. \& Waterman, P.G. (1987). A critical analysis of techniques for measuring tannins in ecological studies I. Techniques dor chemically defining tannins. Oecologia, 72, 137-147. 10.1007/BF00385058

Morais, L. de C. \& Pinheiro, S. S. (2018). Nutrients and bioactive compounds of açaí, bacuri, buriti, camu-camu and cubiu. Tropical fruits-from cultivation to consumption and health benefits (Editors: TODOROV, SD \& PIERI, FA), Nova Science Publisher, Inc, 121-132.

Navarro, F. F., et al. (2015). Quality control and phytochemical screening of the plant drug in the leaves of Morus nigra L. (MORACEAE). Journal of Basic and Applied Pharmaceutical Sciences, 36 (2), 259-265.

Neves L.C., et al. (2015). Bioactive compounds and antioxidant activity in pre-harvest camu-camu [Myrciaria dubia (H.B.K.) Mc Vaugh] fruits. Sci. Horticulturae, 186, 223- 229. 10.1016/j.scienta.2015.02.031

Oliveira, V. B., et al. (2016). Efeito de diferentes técnicas extrativas no rendimento, atividade antioxidante, doseamentos totais e no perfil por clae-dad de dicksonia sellowiana (presl.). Hook, dicksoniaceae. Revista Brasileira de Plantas Medicinais, 18, 230-239. 10.1590/1983-084X/15_106

Park, Y. W. (1996). Determination of moisture and ash contents of food. In: Nollet, L. M. L. Handbook of food analysis: physical characterization and nutrient analysis. Marcel Dekker, 3, 59-92.

Rice-Evans, C. A. \& Miller, N. J. \& Paganga, G. (1996). Structure-antioxidant activity relationships of flavonoids and phenolic acids. Free Radical Biology and Medicine, Orland, 20 (7), 933-956. 10.1016/0891-5849(95)02227-9

Rodrigues, R. B. \& Marx, F. (2006). Camu camu [Myrciaria dubia (HBK) Mc Vaugh]: a promising fruit from the Amazon Basin. NUTRITION-VIENNA, 30 (9), 376.

Rufino, M. D. S. M. et al. (2007). Metodologia científica: determinação da atividade antioxidante total em frutas pela captura do radical livre DPPH. Embrapa Agroindústria Tropical-Comunicado Técnico (INFOTECA-E).

Rufino, M. D. S. M., et al. (2010). "Bioactive compounds and antioxidant capacities of 18 nontraditional tropical fruits from Brazil." Food Chemistry 121(4), 996-1002. 10.1016/j.foodchem.2010.01.037

Sánchez - Moreno, C. \& Larrauri, J. A. \& Saura-Calixto, F. (1998). Um procedimento para medir a eficiência anti-radicalar de polifenóis. Jornal da Ciência da Alimentação e Agricultura, 76 (2), 270-276. 10.1002/(SICI)1097-0010(199802)76:2<270::AID-JSFA945>3.0.CO;2-9

Santos, I. L., et al. (2021). Camu-camu [Myrciaria dubia (HBK) McVaugh]: a review of properties and proposals of products for integral valorization of raw material. Food Chemistry, 131-290. 10.1016/j.foodchem.2021.131290

Schimidt P. C. (1989). Phytopharmaceutical Technology. CRC.

Sharapin, N. (2000). Fundamentos de Tecnologia de Produtos Fitoterápicos. Santa Fé de Bogotá-Colombia:CYTED, 145-157.

Tanaka, O., et al. (1996). Application of saponins in foods and cosmetics: Saponins of Mohave yucca and Sapindus mukurossi. Saponins used in Food and Agriculture, 1-11. 10.1007/978-1-4613-0413-5_1

Teixeira, A. S. \& Chaves, L. Da S. \& Yuyama, K. (2004). Esterases no exame da estrutura populacional de Camu-camu (Myrciaria dubia (Kunth) McVaughMyrtaceae). Acta Amazonica, Manaus, 34 (1). 10.1590/S0044-59672004000100011

Tiwari, P., et al. (2011). Phytochemical screening and Extraction: A Review. Internationale Pharmaceutica Sciencia, 1 (1), 98-106.

Solis V. S., et al. (2009). Evaluacion de la actividad antioxidante de la pulpa, cascara y semilla del fruto del camu camu (Myrciaria dubia H.B.K.). Revista Sociedad Química Perú. 75, 293-299.

Velioglu, Y. S. (1998). Antioxidant activity and total phenolic in selected fruits, vegetables, and grain products. J. Agric. Food Chem., $46,4113-4117$. $10.1021 / \mathrm{j} 9801973$

Villanueva-Tiburcio, J. E. \& Condezo-Hoyos, L. A. \& Asquieri, E. R. (2010). Anthocyanins, ascorbic acid, total polyphenols and antioxidant activity in camucamu (Myrciaria dubia (HBK) McVaugh) peels. Ciênc. Tecnol. Aliment. 30, 151-160. 10.1590/s0101-20612010000500023

Who. (2011). Quality control methods for herbal materials. World Health Organization, 173.

Willerding, A. L., et al. (2020). Estratégias para o desenvolvimento da bioeconomia no estado do Amazonas. Estud. av., São Paulo, 34 (98), 145-166. $10.1590 / \mathrm{s} 0103-4014.2020 .3498 .010$

Yuyama, K. \& Aguiar, J. P. L. \& Yuyama, L. K. O. (2002). Camu-camu: uma fruta fantástica como fonte de vitamina C1. Acta Amazonica, 32 , 169-174. $10.1590 / 1809-43922002321174$ 
Research, Society and Development, v. 11, n. 2, e5811225130, 2022

(CC BY 4.0) | ISSN 2525-3409 | DOI: http://dx.doi.org/10.33448/rsd-v11i2.25130

Zanatta, C. F. \& Mercadante, A. (2007). Composição da fruta tropical brasileira camu-camu (Myrciaria dubia). Food Chemistry, 101 (4), $1526-1532$. 10.1016/j.foodchem.2006.04.004

Zapata, S. M. \& Dufour, J. P. (1993). "Camu-camu Myrciaria dubia (HBK) McVaugh: Chemical composition of fruit." Journal of the Science of Food and Agriculture, 61(3), 349-351. 10.1002/jsfa.2740610310

Zeng, M., et al. (2007). Microscopic examination of changes of plant cell structure in corn stover due to hot water pretreatment and enzymatic hydrolysis. Biotechnology and Bioengineering, 97, 265- 278. 10.1002/bit.21298 